\title{
The Physicochemical and Structural Characteristics of Cultivated Sahlep
}

\author{
Abdullah KURT ${ }^{* 1,2}$, Talip KAHYAOGLU ${ }^{3}$ \\ ${ }^{1}$ Department of Food Engineering, Engineering Architecture Faculty, Bitlis Eren University, 13000 Bitlis, Turkey \\ 2 Department of Food Engineering, Engineering Faculty, Ondokuz Mayıs University, 55139, Samsun, Turkey \\ ${ }^{3}$ Department of Food Engineering, Chemistry-Metallurgical Faculty, Y1ldı Technical University, 34210 Istanbul, \\ Turkey
}

Received: 05 May 2017 - Revised: 12 October 2017 Accepted: 11 November 2017

\begin{abstract}
Sahlep is obtained from tubers of orchids which grown in Mediterranean countries, especially in Turkey. As hydrocolloid, it is used for preparing hot drink and ice cream. However, orchid plants are damaged and sold with high priced. Therefore, searching of alternative production methods of sahlep are in progress. The products of the project, growing possibilities of sahlep orchids at farm conditions (cultivation) were researched in this study. The naturally grown sahlep which belongs to Serapias vomeracea and Orchis sancta species compared with that of cultivated products. Physicochemical and structural characterization analyses were performed for the samples. The tubers were obtained from Serapias vomeracea exhibited higher similarity in physicochemical properties with naturally grown one than Orchis sancta. Samples have Newtonian flow behavior. FTIR experiments which provide knowledge about chemicals bonds in structure revealed that cultivation studies have no effect on sahlep chemical structure. Amorph structure was determined with the XRD experiment for all samples. The variation of surface morphology of sahlep was observed with the cultivation by SEM. DSC and TGA analysis exhibited that samples had similar thermal stabilities. The results obtained showed that sahlep with the same physicochemical and structural characteristics with naturally grown could be achieved by cultivation. The development and widening in this production method provides both protection of wild orchids and cheaper sahlep production.
\end{abstract}

Keywords: Sahlep, Cultivation, Rheology, Characterization

\section{INTRODUCTION}

Sahlep is the roots or tubers of certain wild terrestrial Orchidaceae species [1]. The tubers of Orchis, Ophyris, Serapias, Platanthera, and Dactylorhiza are called as sahlep [2]. It is largely collected in Eastern Mediterranean countries and produced on an average 20 tons in Turkey every year [3]. The tubers of naturally grown orchids are boiled in water to inhibit the enzymatic activity and reduce the loss of water-soluble ingredients and then, dried and ground to produce sahlep powder.

Glucomannan is the most important polysaccharide constituent in sahlep as reserve material due to its stabilizing function and used as low-calorie dietary product. Glucomannans are also considered as water-soluble fibers, which help to normalize blood sugar, relieve stress on the pancreas, and discourage blood sugar abnormalities, such as hypoglycemia. They also exhibited preventative effect on chronic disease and act as a weight control agent [4].

\footnotetext{
*Corresponding Author E-mail: akurt@beu.edu.tr
} 
Sahlep is commonly used as a traditional beverage and a stabilizer for hard serve ice cream. In order to obtain one kilogram of dry sahlep flour, approximately 2600 orchids are needed. Meanwhile 40 different types of 30 million orchids are harvested in Turkey annually. Therefore Turkish Ministry of Agriculture and Rural Affairs in 2003 prohibited to collect the tubers of sahlep [2].

Alternative stabilizers to sahlep have been searched due to its high retailing prices and the danger posed by the possibility of the extinction of Orchids plants. On the other hand, sahlep consumption is not given up easily due to its characteristic flavor in ice creams [5]. Researches about the methods for producing sahlep orchids have been proceed up to now. The favorable development was observed in Turkey about culturing sahlep tubers by growing them in farm conditions. 3 or 5 tubers were collected from each pricked tuber a year later, indicated the high efficiency of the method (Fig. 1). The project was conducted by Aegean Agricultural Research Institute (Menemen/İzmir). The patent of this method were obtained by Tutar and Kanbur [6]. The present study was undertaken to determine to physicochemical and structural characteristics of cultivated sahlep in order to compare their quality with the naturally grown sahlep.

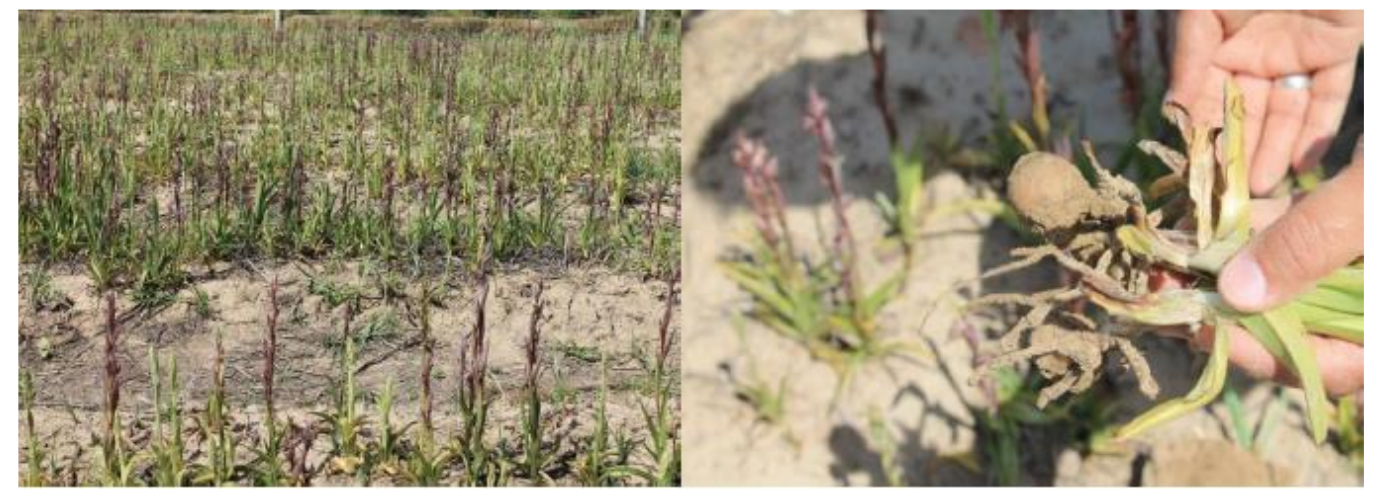

Figure 1. The blooming of sahlep tubers (on the left) and the tubers (on the right).

\section{MATERIAL and METHODS}

\subsection{Material}

Sahlep powders which were the first products of project were obtained from Aegean Agricultural Research Institute in 2013. Tubers of both cultivated and naturally grown Serapias vomeracea $(\mathrm{SV})$ and Orchis sancta $(\mathrm{OS})$ species were boiled in water $15 \mathrm{~min}$ and dried $50{ }^{\circ} \mathrm{C}$ until they had hardened and then ground to obtained sahlep powder. These pretreatments were done by supplier. Samples were coded as SVC- OSC for cultivated and SVN-OSN for naturally grown sahlep.

\subsection{Physicochemical Analysis}

The moisture and ash content were analyzed by using gravimetric methods at 105 and $500{ }^{\circ} \mathrm{C}$, respectively[7]. The elemental analysis was performed by using a Flash $2000 \mathrm{CHNS} / \mathrm{O}$ Analyzers (ThermoFisher Scientific, USA) [8]. The carbon $(\mathrm{C})$, hydrogen $(\mathrm{H})$, nitrogen $(\mathrm{N})$ and sulfur (S) contents were calculated. The protein content $(\%)$ was calculated by multiplying the nitrogen content (\%) by 5.7 [9] The cellulose and lignin contents of samples were determined by the methods proposed by Slavutsky and Bertuzzi [10] using a Fiber Analyzer (ANKOM Technology Fiber Analyzer Model 220, USA). In this method hemicellulose contents were also determined which indicated the glucomannan content of samples. Starch contents of samples were determined by using total starch assay kit (Megazyme)[11]. $\mathrm{pH}$ values of 1\% sahlep solutions were measured with $\mathrm{pH}$ meter (Eutech Instruments, $\mathrm{pH} 700$, Singapore) at room 
temperature. The $L^{*}$-value (lightness), $a^{*}$-value (redness/greenness) and $b^{*}$-values (yellowness/blueness) were determined using a Colorflex, EZ (Hunter associates laboratory, USA). Whiteness index values (WI) of the sahlep powder were also determined as follows [12].

$$
W I=100-\sqrt{\left(100-L^{*}\right)^{2}+\left(a^{*}\right)^{2}+\left(b^{*}\right)^{2}}
$$

\subsection{Molecular Weight Determination}

Molecular weight distribution was determined by high performance size-exclusion chromatography (HPSEC) system (Agilent Technologies, 1100/1200, Australia) with multiple detectors: a UV detector (Agilent 1200), a refractive index (RI) detector (Agilent 1100/1200) and the detector were calibrated using pullulan samples as standards and water was used as a solvent. The flow rate was $0.5 \mathrm{~mL} / \mathrm{min}$ and the columns and detectors were maintained at 35 ${ }^{\circ} \mathrm{C}$.

\subsection{Rheological Analyses}

Analyses of the rheological properties of sahlep solutions at 2, 3 and $4 \%$ concentration were performed using rheometer (HAAKE Mars III; Thermo Scientific, Germany) equipped with a Peltier heating system in a cone and plate configuration (diameter: $35 \mathrm{~mm}$, cone angle: $2^{\circ}$, gap size: $0.105 \mathrm{~mm}$ ). Applied shear rate was within the range of 10-100 1/s, and each step (multiples of $10 \mathrm{~s}^{-1}$ ) lasted 10 seconds at $25^{\circ} \mathrm{C}$.

\subsection{Fourier-transform Infrared Spectroscopy (FTIR)}

The FT-IR spectra of samples were recorded on a spectrophotometer (Perkin Elmer, Model Spectrum Two, Ohio, USA) fit with a Miracle Single-Reflection Diamond ATR device in the wavelength range of $4000-650 \mathrm{~cm}^{-1}$ with a spectral resolution of $4 \mathrm{~cm}^{-1}$.

\subsection{X-ray Diffraction (XRD)}

XRD patterns of samples were obtained using a Rigaku SmartLab X-ray diffractometer $(40 \mathrm{kV}, 30 \mathrm{~mA})$ with a scanning rate of $2^{\circ} / \mathrm{min}$ from $5^{\circ}$ to $45^{\circ}$ ( $2 \theta$ range).

\subsection{Scanning Electron Microscopy (SEM)}

To study the surface characteristics of sahlep samples, the SEM photomicrographs were recorded by using a scanning electron microscope (JSM-7001F, JEOL, Japan). The samples were coated with gold-palladium (Quorum SC7620, England) before the observation under the microscope.

\subsection{Particle Size Measurements}

The mean particle sizes of samples were determined by using a NP size analyzer (Nano ZSP, Malvern Instruments Corp., Worcestershire, U.K.). Dynamic light scattering (DLS) was used to determine the average particle diameter and measurements were done at $173^{\circ}$.

\subsection{Differential Scanning Calorimetry (DSC)}

The thermal analyses of samples were carried out with a model DSC 4000 (Perkin Elmer, USA). The DSC was calibrated with indium (melting point $=156.6{ }^{\circ} \mathrm{C}, \Delta \mathrm{H}=28.5 \mathrm{~J} / \mathrm{g}$ ). $5 \mathrm{mg}$ of the sample in aluminum pan with an empty reference pan were scanned under nitrogen atmosphere $(20 \mathrm{~mL} / \mathrm{min})$ at a heating rate of $10{ }^{\circ} \mathrm{C} / \mathrm{min}$ in a temperature range of $0-350{ }^{\circ} \mathrm{C}$.

\subsection{Thermogravimetric Analysis (TGA)}

TGA measurements were carried out in nitrogen atmosphere $(100 \mathrm{~mL} / \mathrm{min})$ using SDTQ600 (TA Instruments, USA). Analyses were started at $20^{\circ} \mathrm{C}$ and continued up to $700{ }^{\circ} \mathrm{C}$, with a linear increase of $10^{\circ} \mathrm{C} / \mathrm{min}$. Samples were weighed (approximately $10 \mathrm{mg}$ ) in alumina pans. 


\subsection{Statistical Analysis}

SPSS software (Version 16.0, SPSS Inc., Chicago, USA) were used to analyze the data. The means were compared by Tukey's test at a 5\% level of significance using analysis of variance (ANOVA).

\section{RESULTS and DISCUSSIONS}

\subsection{Physicochemical Analysis and Molecular Weights}

Chemical compositions of sahlep flours were presented in Table 1. Dry matter contents of samples varied between $90-91 \%$ ( $p>0.05$ ). Samples exposed to similar pretreatment had suitable moisture for longer storage which should be lower than 10\% [13]. Different geographic region conditions have also effect on moisture content of samples because different moisture contents were reported previously for sahlep [4]. From the results in Table 1, it can be seen that SVN and OSN samples had similar protein content ( $p>0.05$ ) however cultivation process did not affect the protein composition of sahlep. The values of protein content (10-12\%) exhibited pretty higher than the results of Tekinsen and Guner [13], who found between 3.16-4.95\% in ten different sahlep species and Farhoosh and Riazi [4], who found 3.09\% for palmate-tuber sahlep and $7.32 \%$ for rounded-tuber sahlep. There were significantly differences between ash contents of samples. Cultivation studies increased ash content of SV but decreased ash value of OS. The values of ash content (3.73-4.42\%) had higher than the reported studies of Kurt and Kahyaoglu [9] as 2.07 and Tekinsen and Guner [13] as 0.9-2.83.

Glucomannan (GM) is considered as the quality parameter of sahlep [9; 13]. Higher GM content indicates higher quality of sahlep. The used raw material (SV and OS) for cultivation had different GM percentage $(\mathrm{p}<0.05)$. Cultivation process decreased GM content of $\mathrm{SV}$ $(p<0.05)$ but had no effect on OS. Therefore it could be said that quality of sahlep was preserved when cultivation was applied to SV. The negative correlation between glucomannan and starch was reported by Kurt and Kahyaoglu [9] and Tekinsen and Guner [13]. OS which had the higher GM content exhibited lower starch content. Cultivation of SV and OS did not significantly change of starch constituents of sahlep.

Table 1. Physicochemical and elemental compositions, $\mathrm{pH}$ and color values of samples

\begin{tabular}{lllll}
\hline & SVN & \multicolumn{1}{c}{ SVC } & OSN & OSC \\
Dry matter (\%) & $90.84 \pm 0.00^{\mathrm{a}}$ & $90.85 \pm 0.07^{\mathrm{a}}$ & $91.05 \pm 0.02^{\mathrm{a}}$ & $90.62 \pm 0.02^{\mathrm{a}}$ \\
Protein (\%) & $11.20 \pm 0.70^{\mathrm{ab}}$ & $10.27 \pm 0.06^{\mathrm{b}}$ & $12.77 \pm 0.50^{\mathrm{a}}$ & $11.91 \pm 0.18^{\mathrm{ab}}$ \\
Ash (\%) & $4.28 \pm 0.01^{\mathrm{b}}$ & $4.42 \pm 0.03^{\mathrm{a}}$ & $4.00 \pm 0.03^{\mathrm{c}}$ & $3.73 \pm 0.02^{\mathrm{d}}$ \\
Glucomannan (\%) & $40.52 \pm 1.98^{\mathrm{b}}$ & $31.04 \pm 1.09^{\mathrm{c}}$ & $50.92 \pm 0.51^{\mathrm{a}}$ & $52.04 \pm 1.15^{\mathrm{a}}$ \\
Starch (\%) & $31.67 \pm 0.94^{\mathrm{a}}$ & $32.69 \pm 0.70^{\mathrm{a}}$ & $23.47 \pm 0.66^{\mathrm{b}}$ & $23.67 \pm 0.45^{\mathrm{b}}$ \\
Cellulose (\%) & $4.05 \pm 0.31^{\mathrm{b}}$ & $5.99 \pm 0.19^{\mathrm{a}}$ & $4.33 \pm 0.38^{\mathrm{bc}}$ & $4.84 \pm 0.20^{\mathrm{c}}$ \\
Lignin (\%) & $0.27 \pm 0.09^{\mathrm{a}}$ & $0.37 \pm 0.04^{\mathrm{a}}$ & $0.40 \pm 0.12^{\mathrm{a}}$ & $0.51 \pm 0.26^{\mathrm{a}}$ \\
Nitrogen (\%) & $1.90 \pm 0.04^{\mathrm{bc}}$ & $1.80 \pm 0.01^{\mathrm{c}}$ & $2.24 \pm 0.08^{\mathrm{a}}$ & $2.08 \pm 0.03^{\mathrm{ab}}$ \\
Carbon (\%) & $38.81 \pm 1.37^{\mathrm{a}}$ & $40.62 \pm 0.17^{\mathrm{a}}$ & $39.85 \pm 1.21^{\mathrm{a}}$ & $39.38 \pm 0.96^{\mathrm{a}}$ \\
Hydrogen (\%) & $5.84 \pm 0.25^{\mathrm{a}}$ & $6.18 \pm 0.07^{\mathrm{a}}$ & $6.06 \pm 0.20^{\mathrm{a}}$ & $5.99 \pm 0.19^{\mathrm{a}}$ \\
pH & $5.77 \pm 0.03^{\mathrm{c}}$ & $5.77 \pm 0.03^{\mathrm{c}}$ & $6.11 \pm 0.04^{\mathrm{a}}$ & $5.90 \pm 0.02^{\mathrm{b}}$ \\
$L^{*}$ & $70.47 \pm 0.00^{\mathrm{b}}$ & $70.41 \pm 0.00^{\mathrm{b}}$ & $75.10 \pm 0.24^{\mathrm{a}}$ & $68.64 \pm 0.06^{\mathrm{c}}$ \\
$a^{*}$ & $5.95 \pm 0.00^{\mathrm{b}}$ & $6.21 \pm 0.00^{\mathrm{c}}$ & $3.93 \pm 0.07^{\mathrm{d}}$ & $6.50 \pm 0.04^{\mathrm{a}}$ \\
$b^{*}$ & $23.32 \pm 0.02^{\mathrm{a}}$ & $23.51 \pm 0.04^{\mathrm{a}}$ & $21.55 \pm 0.26^{\mathrm{b}}$ & $23.65 \pm 0.02^{\mathrm{a}}$ \\
Whiteness index (WI) & $61.90 \pm 0.01^{\mathrm{b}}$ & $61.69 \pm 0.02^{\mathrm{b}}$ & $66.83 \pm 0.36^{\mathrm{a}}$ & $60.18 \pm 0.05^{\mathrm{c}}$ \\
\hline
\end{tabular}

Values with the different letter within the same line are significantly different $(\mathrm{p}<0.05)$. Results are mean $\pm \mathrm{SD}$ of three determinations.

Cultivation process increased the cellulose content of SV but had no effect on OS. There were no significantly differences between lignin values of samples. The elemental analysis indicated that cultivation process did not change nitrogen, hydrogen and carbon values $(p>0.05)$ 
of SV and OS. Higher nitrogen content of OS was preserved with cultivation at the same soil characteristics. In used sahlep species, OS had higher $\mathrm{pH}$ value. $\mathrm{pH}$ values of OS decreased but $\mathrm{pH}$ stability was observed for SV by cultivation.

Color parameters and whiteness index of sahlep powder were also determined. Naturally obtained OS had the highest lightness value than SV and this property was not changed for SV however $L^{*}$ values decreased for OS. $a^{*}$ values increased both sahlep species with cultivation however $b^{*}$ values decreased for OS and was not change for SV. In order to obtain clearer solution during mix preparation whiteness is important sensorial characteristics. Therefore whiteness index (WI) values were calculated with considering all color parameters [12]. OS which had the higher WI values than SV lost its property after cultivation however SV was preserved its characteristics.

Molecular weight is important parameter for polymer characterization because functional characteristics are related to this. Molecular weight distribution statement was used widely for polymeric systems. Molecular weights of samples were determined as $8.91 \times 10^{5}, 8.83 \times 10^{5}$, $11.23 \times 10^{5}$ and $5.5 \times 10^{5}$ Da for SVN, SVC, OSN and OSC, respectively. It was clear that two different samples which had different molecular weight were used in this study. The sample which had the higher molecular weight (OS) revealed lower molecular weight by cultivation but SV preserved its molecular weight value after cultivation. The results determined in this part indicated that SVC exhibited higher similarity to the naturally obtained one when we compared with OSC. Therefore, we could conclude that SV is more suitable for cultivation.

\subsection{Rheological Analyses}

Viscosity values of both cultivated sahlep samples (SVC and OSC) and naturally obtained samples (SVN and SVC) were presented at Fig.2. It is clear that the values for viscosity followed an increasing trend as the concentration increases. More solid content causes an increase in viscosity as a result of the occurrence of multiple association points among the gum molecules, especially in linear and/or stiff molecules with a large hydrodynamic size $[14 ; 15]$ and can also improve water binding capacity $[16 ; 17]$.

The apparent viscosities of all samples remain stable with increasing shear rate at all concentration; this behavior is the evidence of Newtonian flow behavior even at $4 \%$ sahlep concentration. When other studies was considered, this was an unexpected result because sahlep solutions generally showed non-Newtonian (shear thinning) properties $[4 ; 5 ; 9 ; 17 ; 18]$. One of the explanations of these results could be lower molecular weight and higher polydispersity index of sample. The results about molecular weight were found lower than reported study of sahlep [8;19]. The other reason could be the higher amount percentage of constituents apart from glucomannan such as protein, starch, ash, cellulose and lignin because possible enhancement of interaction between glucomannan chains was hindered by these impurities. Higher protein and ash contents for this work than reported studies were mentioned above. The main impurity is starch which affects the quality of sahlep such as reducing viscosity [20]. Higher glucomannan contents and glucomannan/starch ratio provides higher viscosities [13]. OS species showed higher viscosities than SV but cultivated OS exhibited the lowest viscosities than all other samples. However, cultivation studies did not affect the viscosity values of SV.

Pseudoplastic behavior is important for preparation of hot beverages and ice cream production. These results revealed that used sahlep for cultivation was not proper for preparing of milk beverage and ice cream production due to the lower viscosity characteristics. Therefore, determination characteristics are important before cultivation studies of sahlep. However, rheology results supported the suitability of SV in contrast with OS. 


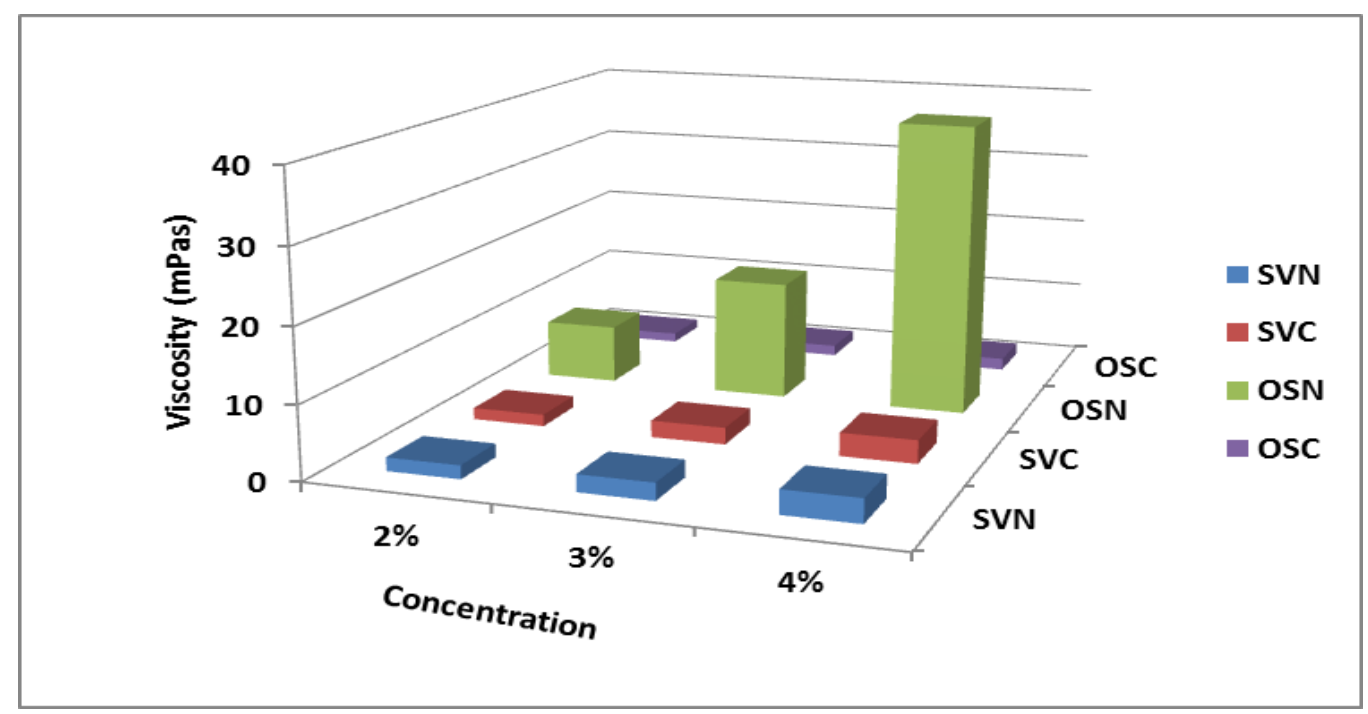

Figure 2. Viscosity of sahlep samples at different concentrations

\subsection{Fourier-transform Infrared Spectroscopy (FTIR)}

Fig. 3 shows the FTIR spectrum of the samples. The broad band between 3500-3000 $\mathrm{cm}^{-1}$ indicated the presence of hydroxyl groups (-OH) due to the moisture and also attributed to the hydroxyl of sugar rings [18]. The peaks in the region $3000-2800 \mathrm{~cm}^{-1}$ is an indicator of $\mathrm{C}-\mathrm{H}$ stretching [9]. The peak at about $1735 \mathrm{~cm}^{-1}$ which was attributed to the presence of acetyl group [8;12]. $1644 \mathrm{~cm}^{-1}$ band attributed to the protein content of samples [11]. The peaks between 800 and $1200 \mathrm{~cm}^{-1}$ demonstrates the finger print region for carbohydrates [21]. The peak about $\beta$-glucosidic and $\beta$-mannosidic linkages was assigned to the 869 and $812 \mathrm{~cm}^{-1}$, respectively. It could be concluded according to these results and compatibility with reported results that cultivation process preserved the chemical structure of sahlep samples.

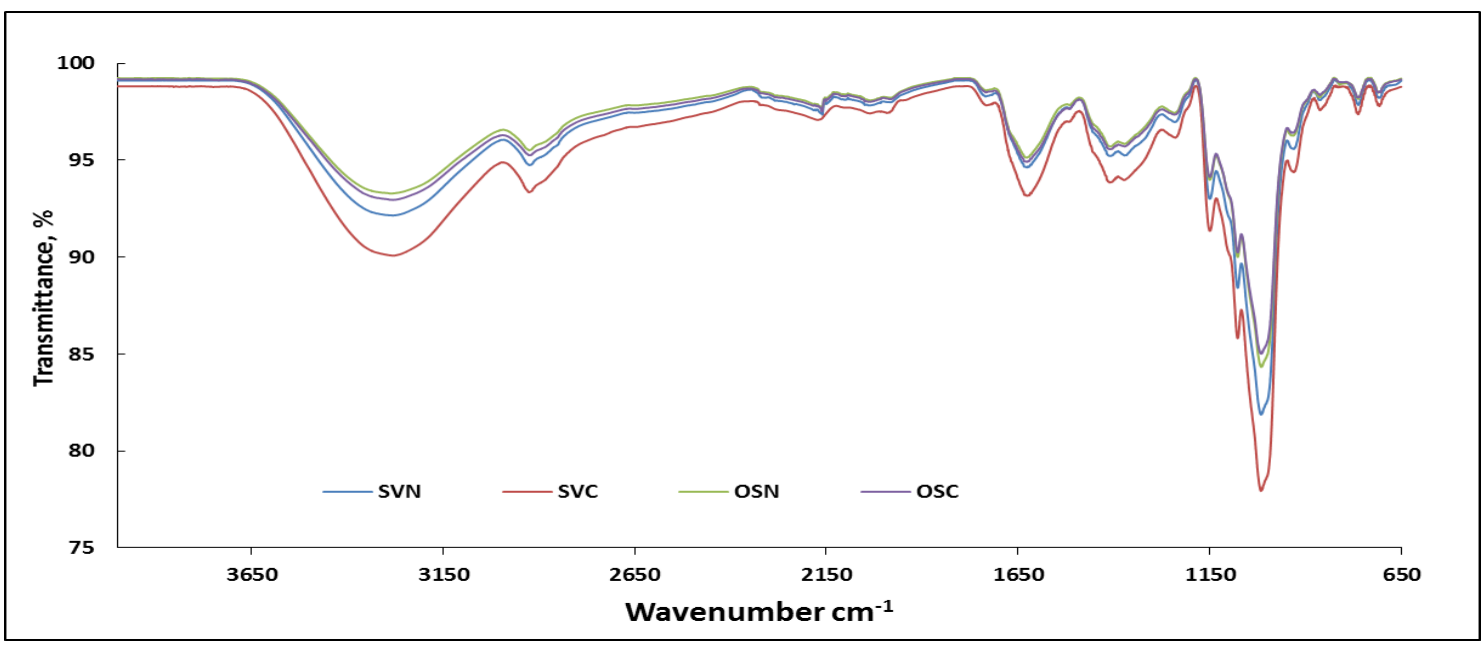

Figure 3. FT-IR spectra of sahlep samples.

\subsection{X-ray Diffraction (XRD)}

X-ray experiments were conducted to determine crystallinity and the effect of cultivation on sahlep samples (Fig.4). Both native and cultivated samples showed a non-crystalline state and broad dispersion peaks, indicating amorphous nature of samples. The broad peak at $2 \theta=$ $20^{\circ}$ is a characteristic peak for sahlep samples which was consistent with the reported result [22]. The sharp peak at $2 \theta=16^{\circ}$ was attributed to the starch contents of samples [23]. This peak 
intensity was consistent with the amount of starch constituents which determined at physicochemical analyses. SV samples included higher starch than OSN and OSV. Amorphous structure was also supported by the thermal analysis (DSC) of samples with the lack of melting peak.

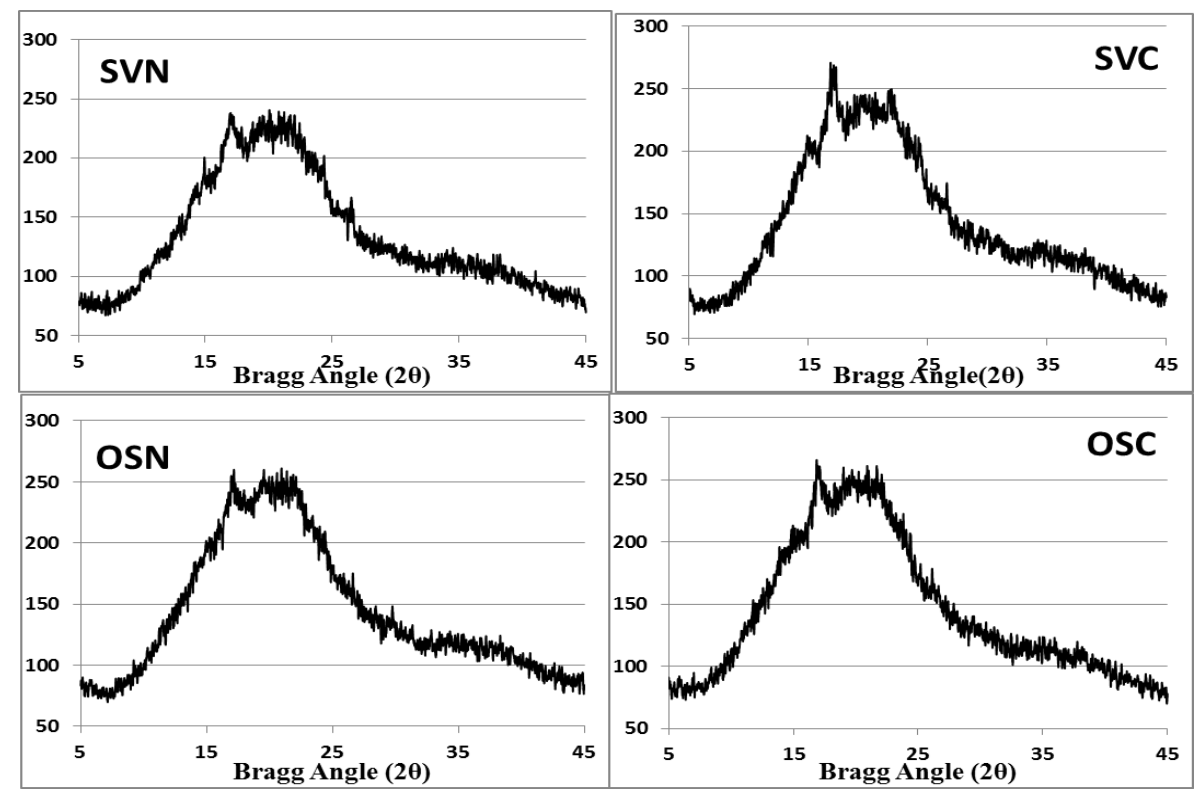

Figure 4. XRD patterns of sahlep samples.

\subsection{Scanning Electron Microscopy (SEM) and Particle Size}

Fig. 5 showed SEM images of sahlep samples. The general shape images were presented with 100x magnification at left side of figure and surface morphology with 2000x at right side. The irregular and round shape of granules were consistent with reported studies [8;9] for sahlep and cultivation process had no effect on this property. On the other hand, both SV samples exhibited smoother surface than OSN and OSC. Rough surface could be attributed to the presence of starch which attached on glucomannan granules [9; 24]. Sizes of particles were determined as 136, 136, 57 and 136 d.nm for SVN, SVC, OSN and OSC, respectively. The lowest particle size was determined for OSN which in the range of reported study for galactomannan [25]. Cultivation study affected particle size of OS but not cause any change on SV. 

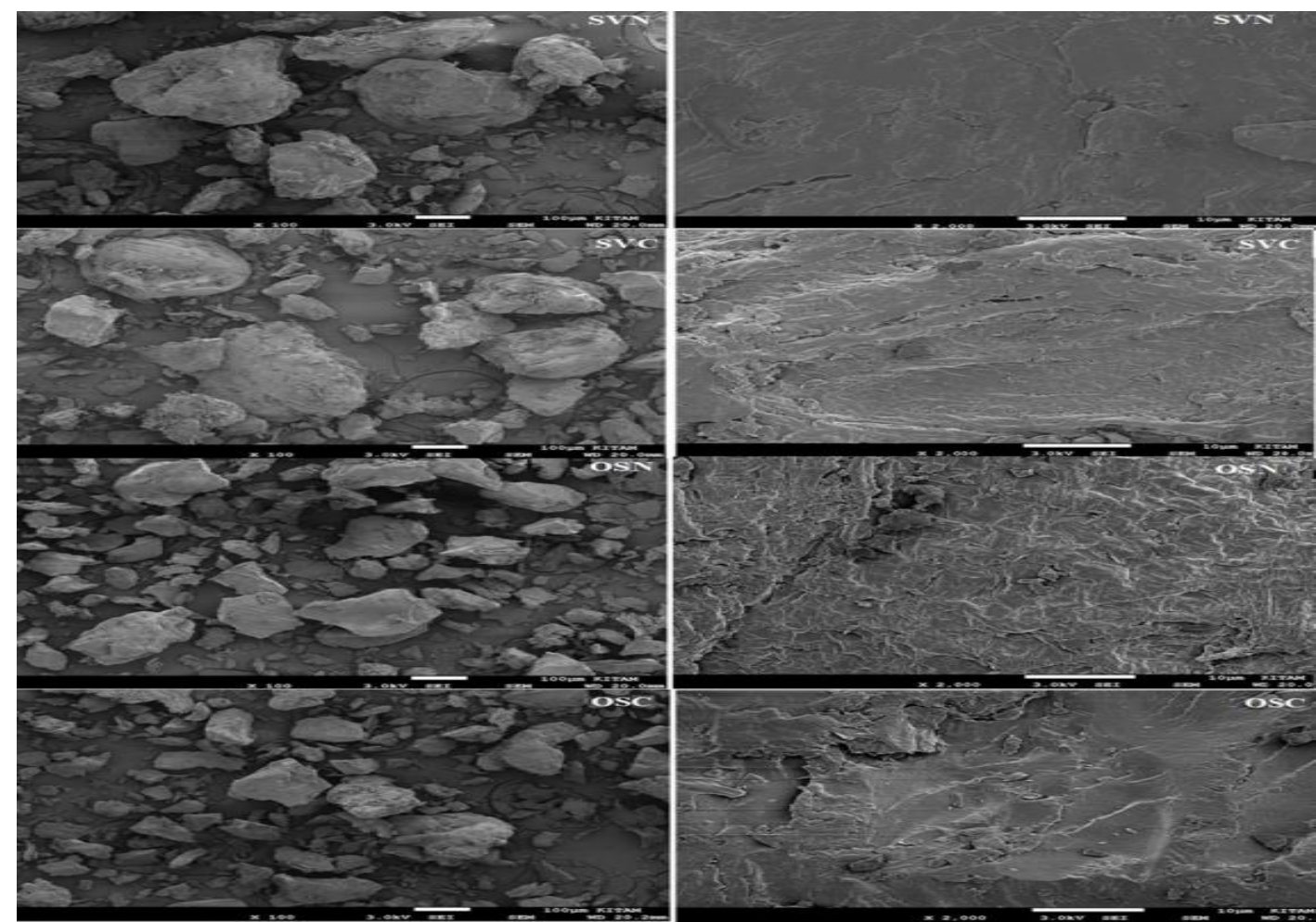

Figure 5. SEM images of sahlep samples.

\subsection{Differential Scanning Calorimetry (DSC)}

The aim of performing DSC analyses is to measure the heat loss or gain due to the physical or chemical changes in sample as a function of temperature. DSC thermograms of sahlep samples were shown in Fig. 6. All samples exhibited two distinct peaks: at approximately $100{ }^{\circ} \mathrm{C}$ was attributed to the loss of water in the samples and exothermic peak that occurred at approximately $300^{\circ} \mathrm{C}$ was defined as the pyrolysis of sahlep by breaking hydrogen bonds in polysaccharides during temperature increments. Higher exothermic temperatures represent the higher thermal stability. The transition at about $70{ }^{\circ} \mathrm{C}$ was attributed to the gelatinization of starch which evaluated at physiochemical and XRD results [17; 26]. All samples had the same thermal properties and cultivation studies had no effect on it.

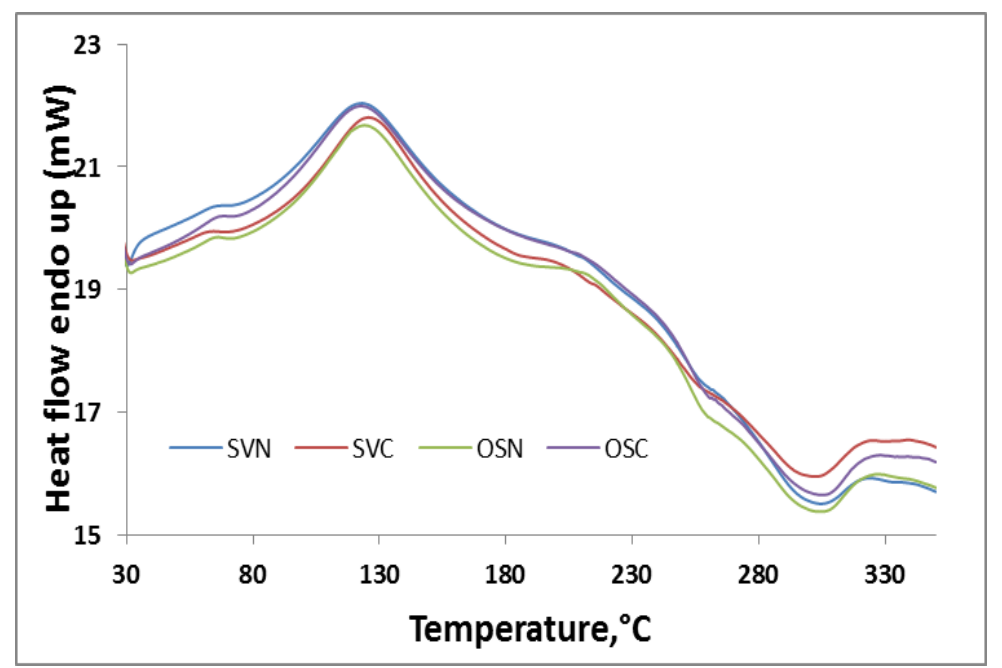

Figure 6. DSC thermograms of sahlep samples 


\subsection{Thermogravimetric Analysis (TGA)}

Thermogravimetric analysis (TGA) was conducted in order to observe cultivation effect on the decomposition pattern and the thermal stability of the samples. Fig. 7 consists of two mass loss events for all samples observed with TGA results. The initial weight loss about 100 ${ }^{\circ} \mathrm{C}$ was due to the evaporation of water [27]. The loss weights of samples were $9-10 \%$ which was consistent with the moisture content of samples. The onset point of second step of samples which were defined as dehydration of the saccharide rings and depolymerization meaning that polysaccharide thermal decomposition determined as $242^{\circ} \mathrm{C}$ and mass loss at this period was $44.95 \%$. These parameters were similar for all samples and cultivation process did not change the thermal properties of sahlep as mentioned at DSC results.

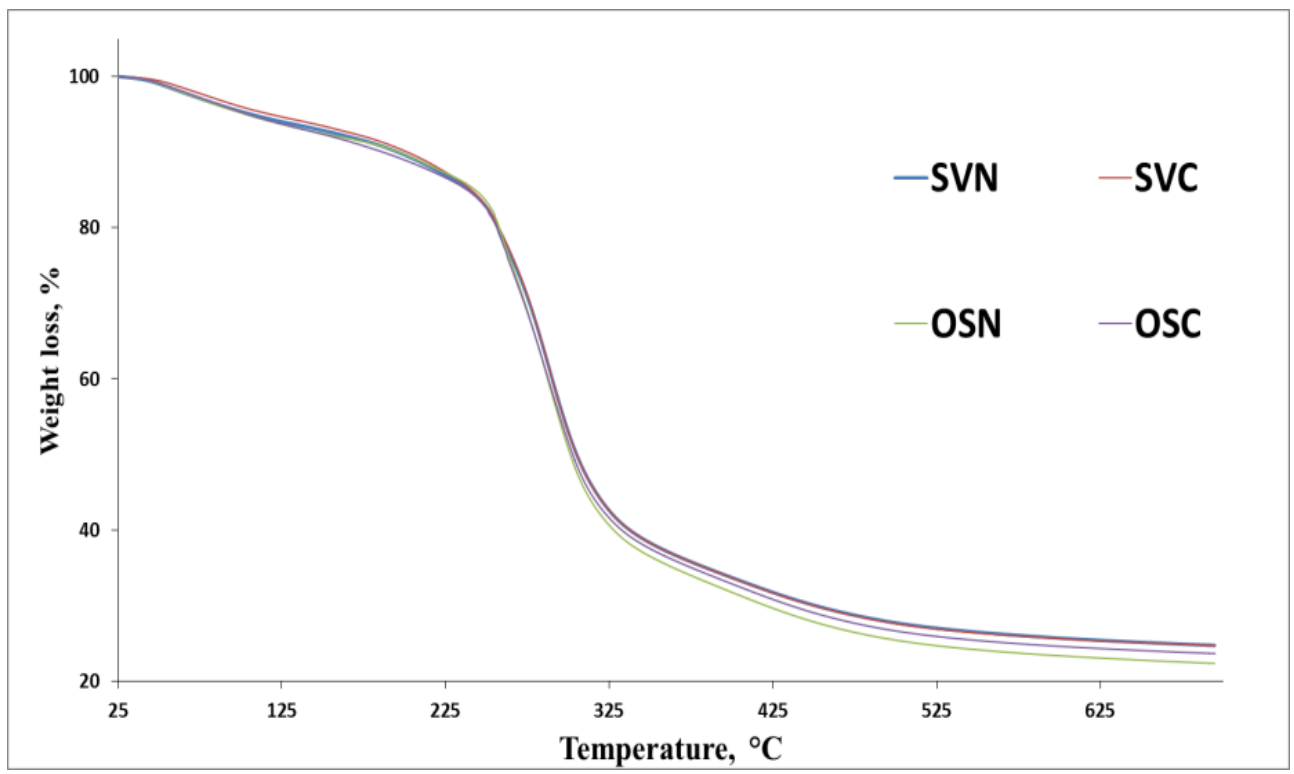

Figure 7. TGA thermograms of sahlep samples.

\section{CONCLUSIONS}

The first products of the project about cultivation of sahlep in farm conditions were characterized in this study. The first thing should be done that higher quality sahlep should be used for cultivation because viscosity which is the main property of sahlep was low. Glucomannan and ash contents were changed based on species. As a result of physicochemical analysis it could be concluded that Serapias vomeracea exhibited higher similarity with the cultivated one than Orchis sancta. Structural characteristics of cultivated samples exhibited same results with naturally obtained sahlep. However, it is clear that species is an important factor. The most suitable species should be determined for studied farm conditions. It is expected that the products of next years should also be determined to observe changing with regard to the years. Apart from these evaluations, this project is the promising and a very important development for both protections of wild orchids and cheaper sahlep production.

\section{Acknowledgement}

This work was supported by Ondokuz Mayis University Research Foundation (PYO.MUH.1901.15.001).

\section{Conflict of Interests}

Authors declare that there is no conflict of interests. 


\section{REFERENCES}

[1] Kaya, S., \& Tekin, A. R. (2001). The effect of sahlep content on the rheological characteristics of a typical ice-cream mix. Journal of Food Engineering, 47(1), 59-62.

[2] Tamer, C. E., Karaman, B., \& Copur, O. T. (2006). A traditional Turkish beverage: Sahlep. Food Reviews International, 22(1), 43-50.

[3] Hossain, M. M. (2011). Therapeutic orchids: traditional uses and recent advances - An overview. Fitoterapia, 82(2), 102-140.

[4] Farhoosh, R., \& Riazi, A. (2007). A compositional study on two current types of sahlep in Iran and their rheological properties as a function of concentration and temperature. Food Hydrocolloids, 21(4), 660-666.

[5] Kurt, A., Cengiz, A., \& Kahyaoglu, T. (2016). The effect of gum tragacanth on the rheological properties of sahlep based ice cream mix. Carbohydrate Polymers, 143, 116123.

[6] Tutar, M., \& Kanbur, M. S. (2013). Orchid comprising several new tubers and the production method thereof: Google Patents.

[7] AOAC. (2005). Official methods of analysis (18 ed.). Washington.

[8] Kurt, A., \& Kahyaoglu, T. (2017). Purification of glucomannan from sahlep: Part 2. Structural characterization. Carbohydrate Polymers, 169, 406-416.

[9] Kurt, A., \& Kahyaoglu, T. (2015). Rheological properties and structural characterization of sahlep improved by ethanol treatment. Carbohydrate Polymers, 133, 654-661.

[10] Slavutsky, A. M., \& Bertuzzi, M. A. (2014). Water barrier properties of starch films reinforced with cellulose nanocrystals obtained from sugarcane bagasse. Carbohydrate Polymers, 110, 53-61.

[11] Chua, M., Chan, K., Hocking, T. J., Williams, P. A., Perry, C. J., \& Baldwin, T. C. (2012). Methodologies for the extraction and analysis of konjac glucomannan from corms of Amorphophallus konjac K. Koch. Carbohydrate Polymers, 87(3), 2202-2210.

[12] Kurt, A., \& Kahyaoglu, T. (2017). Gelation and structural characteristics of deacetylated sahlep glucomannan. Food Hydrocolloids, 69, 255-263.

[13] Tekinsen, K. K., \& Guner, A. (2010). Chemical composition and physicochemical properties of tubera sahlep produced from some Orchidaceae species. Food Chemistry, $121(2), 468-471$.

[14] Lin, H., \& Huang, A. S. (1993). Chemical-Composition and Some Physical-Properties of a Water-Soluble Gum in Taro (Colocasia-Esculenta). Food Chemistry, 48(4), 403-409.

[15] Vardhanabhuti, B., \& Ikeda, S. (2006). Isolation and characterization of hydrocolloids from monoi (Cissampelos pareira) leaves. Food Hydrocolloids, 20(6), 885-891.

[16] Gómez-Díaz, D., \& Navaza, J. M. (2003). Rheology of aqueous solutions of food additives: Effect of concentration, temperature and blending. Journal of Food Engineering, 56(4), 387-392.

[17] Kurt, A., \& Kahyaoglu, T. (2017). Purification of glucomannan from sahlep: Part 1. Detailed rheological characteristics. Carbohydrate Polymers, 168, 138-146.

[18] Kurt, A., \& Kahyaoglu, T. (2014). Characterization of a new biodegradable edible film made from sahlep glucomannan. Carbohydrate Polymers, 104, 50-58.

[19] Pourjavadi, A., Fakoorpoor, S. M., \& Hosseini, S. H. (2013). Novel cationic-modified sahlep as an efficient flocculating agent for settling of cement slurries. Carbohydrate Polymers, 93(2), 506-511. 
[20] Yoshimura, M., Takaya, T., \& Nishinari, K. (1996). Effects of konjac-glucomannan on the gelatinization and retrogradation of corn starch as determined by rheology and differential scanning calorimetry. Journal of Agricultural and Food Chemistry, 44(10), 2970-2976.

[21] Nep, E. I., \& Conway, B. R. (2011). Physicochemical characterization of grewia polysaccharide gum: Effect of drying method. Carbohydrate Polymers, 84(1), 446-453.

[22] Razavi, M., Nyamathulla, S., Karimian, H., \& Noordin, M. I. (2014). Novel swellable polymer of orchidaceae family for gastroretentive drug delivery of famotidine. Drug Design Development and Therapy, 8, 1315-1329.

[23] Liu, Y., \& Ng, P. K. W. (2015). Isolation and characterization of wheat bran starch and endosperm starch of selected soft wheats grown in Michigan and comparison of their physicochemical properties. Food Chemistry, 176(0), 137-144.

[24] Xu, W., Wang, S., Ye, T., Jin, W., Liu, J., Lei, J., et al. (2014). A simple and feasible approach to purify konjac glucomannan from konjac flour - Temperature effect. Food Chemistry, 158, 171-176.

[25] Haddarah, A., Bassal, A., Ismail, A., Gaiani, C., Ioannou, I., Charbonnel, C., et al. (2014). The structural characteristics and rheological properties of Lebanese locust bean gum. Journal of Food Engineering, 120, 204-214.

[26] Li, J., Ye, T., Wu, X., Chen, J., Wang, S., Lin, L., et al. (2014). Preparation and characterization of heterogeneous deacetylated konjac glucomannan. Food Hydrocolloids, 40, 9-15.

[27] Razmkhah, S., Mohammadifar, M. A., Razavi, S. M. A., \& Ale, M. T. (2016). Purification of cress seed (Lepidium sativum) gum: Physicochemical characterization and functional properties. Carbohydrate Polymers, 141, 166-174. 